Laparoscopia y Robótica

Arch. Esp. Urol., 61, 3 (397-400), 2008

\title{
TRATAMIENTO LAPAROSCÓPICO DE QUISTES RENALES SIMPLES SINTOMÁTICOS
}

\author{
Octavio A. Castillo, Diego DeGiovanni, Rafael Sánchez-Salas, Alejandro Foneron, Gonzalo \\ Vitagliano, Manuel A. Díaz y Marcelo Fajardo.
}

Sección de Endourología y Laparoscopia Urológica. Clínica Santa María.

Departamento de Urología. Facultad de Medicina. Universidad de Chile. Santiago de Chile. Chile.

\begin{abstract}
Resumen.- OBJETIVO: Los Quistes Renales Simples (QRS) son, en general, asintomáticos y no requieren tratamiento. Para los QRS que ameritan terapéutica quirúrgica, la vía laparoscópica ofrece una opción mínimamente invasiva. El objetivo del presente trabajo es mostrar los resultados obtenidos con esta técnica quirúrgica en nuestra institución.
\end{abstract}

MÉTODOS: Se analizaron en forma retrospectiva 41 casos de pacientes con QRS sintomáticos que fueron tratados mediante marsupialización laparoscópica; todas realizadas por el mismo equipo quirúrgico entre noviembre de 1992 y mayo de 2006. Las vías de abordaje utilizadas fueron la endoscópica transperitoneal y retroperitoneal. Se registraron datos demográficos y perioperatorios de forma prospectiva en una base de datos.

RESULTADOS: En el período comprendido entre noviembre de 1992 y mayo de 2006, 41 pacientes fueron operados con el diagnóstico de QRS. La serie estaba formada por 23 (56\%) mujeres y 18 (44\%) hombres con una edad promedio de 54 años (rango: 27-74 años). Todos los pacientes tratados presentaban síntomas, siendo el más frecuente dolor. El tamaño promedio de las lesiones fue de $10 \mathrm{~cm}$. (rango: $5-16 \mathrm{~cm}$.). Se realizó abordaje transperitoneal en 24 pacientes (58\%) y retroperitoneal en 17 (42\%). Dieciséis quistes se localizaron en el riñón izquierdo (39\%), 24 en el derecho (54\%) y un caso era bilateral (3\%). El tiempo operatorio promedio fue de $52 \mathrm{~min}$. (rango: 20-150 min.), la estadía hospitalaria fue de 42 horas (rango: $12-96$ h.). Treinta y nueve de las lesiones (95.2\%) correspondieron al tipo Bosniak I y 2 (4.8\%) al tipo Bosniak II. La patología final confirmó QRS en todas las lesiones. Se presentó una única recidiva que fue resuelta con tratamiento percutáneo.

CONCLUSIÓN: La marsupialización laparoscópica es una excelente opción quirúrgica para el tratamiento de los QRS sintomáticos, siendo este tratamiento realizable y seguro.

Palabras clave: Laparoscopia. Quiste renal.

Summary.- OBJECTIVES: Simple renal cysts (SRC) are usually asymptomatic and do not require any kind of treatment. Laparoscopy is a reasonable minimally invasive surgical option for symptomatic cysts. Herein, we present the results of the laparoscopic technique for the treatment of symptomatic renal cysts performed in our

\section{institution.}

Octavio Castillo

Departmento de Urología

$\frac{0}{u}$
$\frac{1}{0}$
0
$\frac{1}{0}$
0
0
0
$\vdots$
0
$U$

Avenida Santa María 0500

7530234 Providencia

Santiago de Chile. (Chile).

octaviocastillo@vtr.net

Trabajo recibido: 14 de mayo 2007 
METHODS: Between November 1992 and May 2006, 41 patients with symptomatic renal cysts were laparoscopically treated. The surgical techniques employed were transperitoneal and retroperitoneal. De-mographic and surgical data was prospectively collec-ted and analysis retrospectively performed.

RESULTS: 41 patients were treated for symptomatic renal cysts in a fourteen year period. $23(56 \%)$ women and 18 (44\%) men, with a median age of 54 years. (Range 27-74 years.). All patients in the series were symptomatic. Pain was the most frequent symptom. Median cyst size was $10 \mathrm{~cm}$. (range $5-16 \mathrm{~cm}$.). Transperitoneal appro-ach was performed in 24 patients (58\%) and retrope-ritoneal in 17 (42\%). No differences were verified between these approaches. 16 lesions were located at the left renal unit, 24 in the right renal unit (54\%) and one case presented bilateral lesions (3\%). Median operative time was $52 \mathrm{~min}$. (range 20-150 min.), me-dian hospital stay was 42 hours (range 12-96 h.). 39 cysts (95.2\%) were classified as Bosniak's type I lesio-ns, 2 lesions (4.8\%) were type II based on Bosniak's description. Final pathology confirmed every lesion as a simple renal cyst. There was only one relapse in the series. The latter was treated percutaneously.

CONCLUSIONS: Laparoscopy is a feasible, safe and advantageous surgical therapeutic option for sympto-matic renal cysts.

Keywords: Renal cyst. Laparoscopy. Treatment.

\section{MATERIAL Y MÉTODOS}

Se evaluaron 41 casos de pacientes portadores de QRS sintomáticos operados por vía laparoscópica entre Mayo de 1992 hasta Junio de 2006, de los cuales $23(56 \%)$ fueron mujeres y 18 (44\%) hombres, con un promedio de edad de 54 años (rango de 27-74 años). Dentro de la sintomatología, el dolor se presentó en todos los pacientes, pero además 6 (14\%) tuvieron aumento del volumen quiste, $2(4.8 \%)$ presentaban compresión del parénquima renal y 2 $(4.8 \%)$ se palpaban como masas abdominales. Todos se diagnosticaron por ecografía o tomografía axial computarizada (TAC) con contraste (Figura 1). Los quistes eran únicos en 38 pacientes $(92 \%)$ y múltiples en 3 de los mismos (8\%). Todas las cirugías fueron realizadas por el mismo equipo quirúrgico. La vía de abordaje laparoscópica utilizada fue la transperitoneal y retroperitoneal. Esta última vía de abordaje se utilizó de forma exclusiva para quistes del polo inferior. El control postoperatorio se realizó a los 3, 12 y 24 meses con exámenes clínicos y ecográficos.

\section{Técnica de marsupialización transperitoneal}

Con anestesia general se ubica al paciente en decúbito lateral opuesto al sitio quirúrgico con elevación de la fosa lumbar. Se coloca sonda nasogástrica. Se crea neumoperitoneo con inserción de aguja de Veress en punto de Mc Burney (derecho o izquierdo). Se introduce el primer trocar de $10 \mathrm{~mm}$ en la región para-umbilical por donde se colocará la óptica de $30^{\circ}$. Un segundo trocar de $10 \mathrm{~mm}$ es

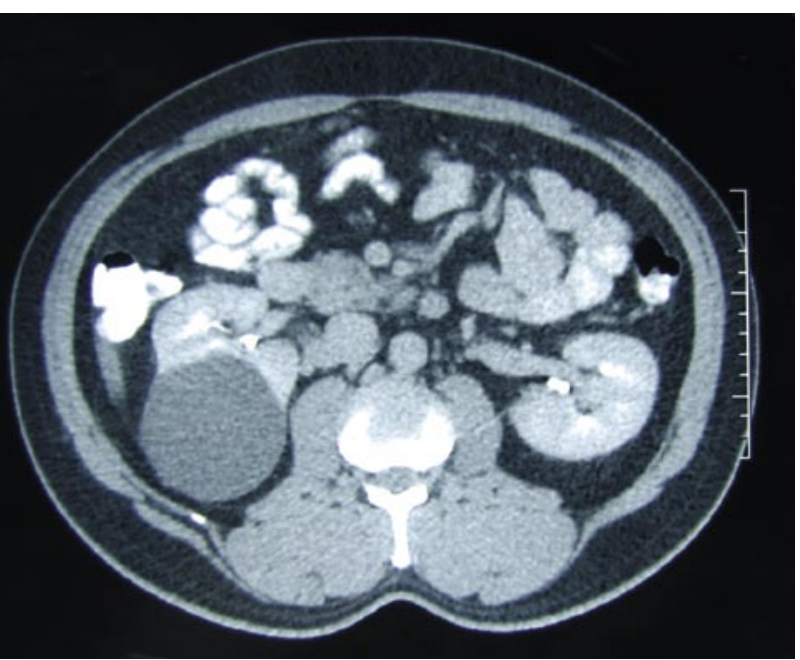

FIGURA 1. Aspecto de un quiste renal simple derecho en una tomografía axial computada con contraste. patología frecuente, con una incidencia que aumenta con la edad (1). A pesar de la existencia de los quistes renales complejos, los QRS son los más habituales (2). Según la clasificación de Bosniak (3), los QRS corresponden al tipo I con riesgo bajo de malignidad. Ellos tienen una evolución generalmente asintomática, sin consecuencias clínicas y su diagnóstico es incidental. Sin embargo, una pequeña proporción de los mismos pueden generar síntomas, siendo el más común es el dolor, aunque también pueden manifestarse con hematuria, hipertensión arterial y obstrucción pielocalicial (2). Para aquellos casos sintomáticos o para los cuales técnicas no quirúrgicas como la inyección de sustancias esclerosantes y/o punciones aspirativas han fracasado, la cirugía laparoscópica emerge como una excelente opción. Este trabajo presenta nuestra experiencia en el tratamiento de quistes simples sintomáticos tratados por abordaje laparoscópico. 
posicionado a $2 \mathrm{~cm}$ subcostal a la altura de la línea medio clavicular bajo visión laparoscópica. El último puerto de $5 \mathrm{~mm}$ es introducido en el sitio de Mc Burney homolateral a la patología. Estos dos últimos bajo visión. Se libera el tejido mesocólico sobre el quiste mediante bisturí armónico para exponer la lesión, sin movilizar el colon. Se punciona el quiste, aspirando su contenido, el que luego se enviará para análisis citológico (Figura 2). Con esta maniobra se limita perfectamente el parénquima renal de la pared del quiste. Se abre el mismo con tijera fría y se reseca

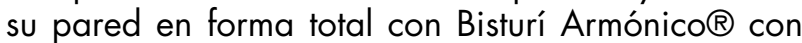
el cuidado necesario para no lesionar parénquima renal sano y evitar así un sangrado del mismo (Figura 3 ). Se controla la hemostasia del lecho del quiste. No se deja drenaje y se retira la sonda nasogástrica colocada previamente.

\section{Técnica de marsupialización retroperitoneal}

Se coloca el paciente en decúbito lateral. Se realiza una incisión de $2 \mathrm{~cm}$. en el vértice de la $12 a$ costilla, se procede a abrir la aponeurosis lumbar y se diseca el espacio retroperitoneal en forma digital. Se coloca un trócar de $10 \mathrm{~mm}$ en la fosa ilíaca y otro de $5 \mathrm{~mm}$ en el ángulo costovertebral. A nivel de la incisión inicial se coloca un trócar de Hasson para la óptica de 30․ El resto de la operación se realiza siguiendo los mismos principios enunciados para la técnica transperitoneal.

\section{RESULTADOS}

Se trataron un total de 41 lesiones quísticas renales sintomáticas. Los estudios de imagen preope-

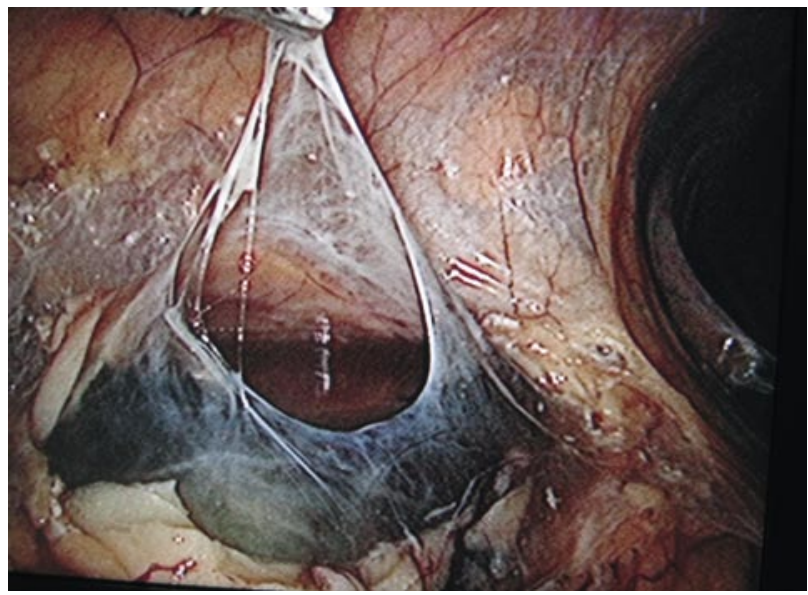

FIGURA 2. Quiste renal simple derecho abierto. Se observa el contenido líquido en su interior, el que fue aspirado parcialmente y enviado a estudio citológico. ratorios permitieron evidenciar las lesiones en 16 unidades renales izquierdas (39\%), 24 unidades derechas $(54 \%)$ y en un caso en ambas unidades renales (3\%). Según la clasificación de Bosniak, 39 (95.2\%) eran tipo I y $2(4.8 \%)$ eran tipo II. Con respecto a la ubicación en el riñón, 16 eran del polo superior $(39 \%), 12$ del inferior $(29 \%), 10$ de la región interpolar $(24 \%)$ y 3 parapiélicos (7\%). El tamaño promedio fue de $10 \mathrm{~cm}$ (rango: $5-16 \mathrm{~cm}$ ).

Todos los pacientes fueron operados por vía laparoscópica. En 24 (58\%) pacientes se utilizo la vía transperitoneal mientras que en $17(42 \%)$ casos se abordó por vía retroperitoneal. El tiempo operatorio promedio fue de 52 minutos (rango: 20-150 minutos). No se utilizaron catéteres ni drenajes. La estadía hospitalaria promedio fue de 42 horas (rango: 12-96 horas). Todos los procedimiento quirúrgicos finalizaron sin necesidad de conversión a cirugía abierta y no hubo complicaciones perioperatorias. El análisis anatomopatológico informó en la totalidad de las muestras quistes renales simples. Tuvimos una sola recidiva $(2.4 \%)$ a los seis meses de la cirugía, con un crecimiento de $4 \mathrm{~cm}$, la cuál se solucionó con tratamiento percutáneo. Todos los pacientes refirieron estar asintomáticos luego del tratamiento y su control se realizó mediante ecografía y evaluación clínica durante 3, 12 y 24 meses. Los pacientes retomaron sus actividades habituales antes de los 10 días posterior a la cirugía

\section{DISCUSIÓN}

Clásicamente los QRS sintomáticos se manejan en primera instancia mediante fármacos analgé-

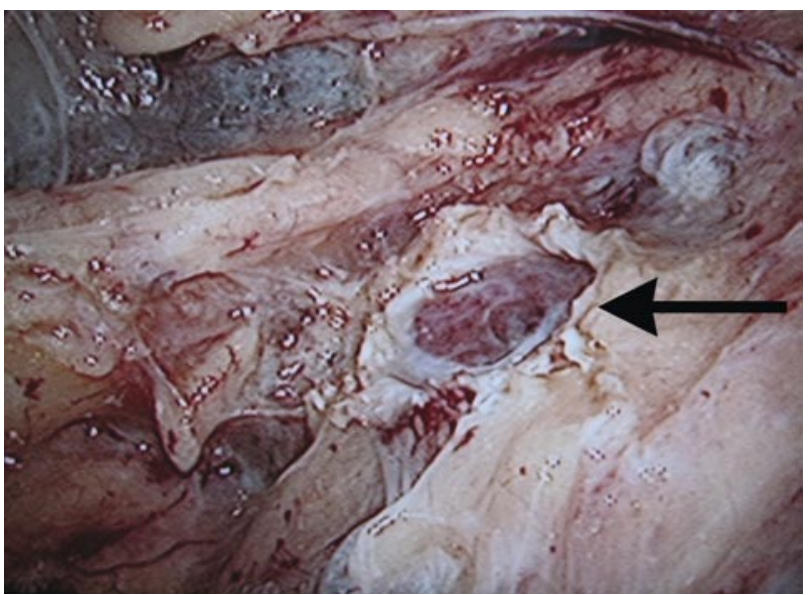

FIGURA 3. Marzupialización finalizada. Se observa el parénquima renal (flecha negra) con una hemostasia satisfactoria. 
sicos no esteroidales o con opiáceos en forma temporal. La segunda línea de tratamiento, cuando la terapia farmacológica es insuficiente, comprende la descompresión de los QRS y la inyección de sustancias esclerosantes por vía percutánea. El abordaje mediante lumbotomía se considera demasiado agresivo para esta patología y encuentra gran resistencia para su aplicación. Es así como en varias series publicadas se han demostrado los pobres resultados de estos abordajes en relación al tiempo de control en relación a la recidiva y el dolor (4). Es por eso que la aparición de la laparoscopia hace revolucionar las estrategias de tratamiento para estos pacientes sintomáticos (5).

Las indicaciones que consideramos para la realización de la cirugía laparoscópica son: dolor, hematuria, hipertensión arterial, masa palpable abdominal, falla en el tratamiento percutáneo con aspiración o inyección de sustancias esclerosantes, o un tamaño del quiste mayor a $8 \mathrm{~cm}$ de diámetro o crecimiento en su control. El abordaje transperitoneal se prefiere para aquellos quistes de localización renal superior y los de ubicación antero-medial cercanos al hilio del riñón. Las lesiones ubicadas en el polo inferior tienen una buena accesibilidad por vía retroperitoneal $(6,7)$. La mayoría de los urólogos prefieren la vía transperitoneal argumentando que a través de la vía lumboscópica el espacio de trabajo es más reducido con menos lugar para la localización de los trócares, visibilidad y orientación (6). Sin embargo el abordaje transperitoneal corre con el peligro de la lesión de órganos abdominales, requiere movilizar el intestino para alcanzar una mejor vía de acceso al quiste y ocasiona en casos íleo paralítico postoperatorios (8). También se puede esparcir el contenido quístico dentro de la cavidad peritoneal, el cual pudiere estar infectado o ser de características neoplásicas. Según los resultados evidenciados en este trabajo, no existen diferencias significativas entre una y otra vía de abordaje. El promedio de tiempo operatorio en nuestra serie fue de 52 minutos y el de la estadía hospitalaria de 42 horas, siendo estos parámetros, menores al de reportes previamente publicados (8 - 13). Nuestro propósito fue mostrar la eficacia de la técnica laparoscópica en términos de mínimas complicaciones, menor tiempo quirúrgico y hospitalario, una rápida recuperación postquirúrgica y buenos resultados demostrables en el tiempo.

\section{CONCLUSIÓN}

Creemos, en base a nuestros resultados, que la vía laparoscópica ha demostrado seguridad y eficacia comparable con la cirugía abierta, en el manejo de los QRS. Se agregan además todos los bene- ficios de la cirugía mínimamente invasiva como son la corta hospitalización, menor dolor postoperatorio, escaso tiempo de recuperación y retorno precoz a las actividades habituales, así como los buenos resultados estéticos. La marsupialización laparoscópica, es además, un procedimiento simple, con pocas complicaciones y mínima tasa de recidivas.

\section{BIBLIOGRAFIA y LECTURAS RECOMENDADAS ( ${ }^{*}$ lectura de interés $y^{* *}$ lectura fundamental)}

**1. NAOKI, T.; KENTARO, I.; YOSUKE, M. y cols.: "The natural history of simple renal cysts." J Urol., 167: 21, 2002.

**2. GLASSBERG, K.: "Renal dysplasia and cystic disease of the kidney." In Walsh P, Retik A Vaughan E, Wein A eds., Campbell's Urology, 7th edn., 1764, WBSaunders, Philadelphia, 1998.

3. BOSNIAK, M.: "The current radiological approach to renal cysts." Radiology.,1, 1989.

4. OkEKE, A.; MITCHLEMORE, A.; KEELEY, F. y cols.: "A comparison of aspiration and sclerotherapy with laparoscopic de-roofing in the management of symptomatic simple renal cysts." BJU Int., 92:610, 2003.

5. WOLF, J. Jr.: "Evaluation and management of solid and cystic renal masses." J Urol.,159:1120, 1998.

**6. DOUMAS, K.; SKREPETIS, K.; LYKOURINAS, M.: "Laparoscopic ablation of symptomatic peripelvic renal cysts." J Endourol.,18:45, 2004.

**7. HoeNIG, D.; MC DOUGALL, E.; SHALVAH, A.y cols.:"Laparoscopic ablation of peripelvic renal cysts." J Urol., 158:1345, 1997.

8. NARMADA, P.; GUPTA, R.; ASHOK, K. y cols.: "Retroperitoneoscopic decortication of symptomatic renal cysts." J Endourol., 19:831,2005.

**9. 9. HEMAL, A.: "Laparoscopic management of renal cystic disease." Urol Clin North Am., 28:115, 2001.

10. DENIS, E.; NICOLAS, F.; BEN RAIS, N. y cols.. "Laparoscopic surgical tratment of simple cysts of kidney." Prog Urol., 8:195,1998.

11. ATUG, F.; BURGESS, S.; RUIZ-DEYA, G. y cols.: "Long term durability of laparoscopic decortication of symptomatic renal cysts." Urology., 68: 272, 2006.

12. HANASH, K.; AL-OTHAMAN, K.; MOKHTAR, A. et al.: "Laparoscopic ablation of giant renal cysts." J Urol., 169; 781, 2001.

13. MC NALLY, M.; ERTURK, E.; OLEYOURRYK, G. et al.: "Laparoscopic cyst decortication using the harmonic scalpel for symptomatic autosomal dominant polycystic kidney disease." J Endourol.,15: 597, 2001. 